\title{
Circuit
}

Musiques contemporaines

\section{Dompter la mer sauvage}

Réflexions sur Evryali de Iannis Xenakis

\section{Taming the Savage Sea}

\section{Marc Couroux}

Volume 5, numéro 2, 1994

Espace Xenakis

URI : https://id.erudit.org/iderudit/902107ar

DOI : https://doi.org/10.7202/902107ar

Aller au sommaire du numéro

Éditeur(s)

Les Presses de l'Université de Montréal

ISSN

1183-1693 (imprimé)

1488-9692 (numérique)

Découvrir la revue

Citer cet article

Couroux, M. (1994). Dompter la mer sauvage : réflexions sur Evryali de Iannis Xenakis. Circuit, 5(2), 55-68. https://doi.org/10.7202/902107ar
Résumé de l'article

Cet article fait état de la problématique des interprètes confrontés à des oeuvres « irréalisables » dans leur intégrité. La question est abordée sous les angles de la technique, de l'esthétique et de l'éthique. Face à diverses solutions proposées, l'interprète doit définir ses choix en s'appuyant sur une analyse de l'oeuvre qui lui permette d'en retrouver le sens. d'utilisation que vous pouvez consulter en ligne.

https://apropos.erudit.org/fr/usagers/politique-dutilisation/ 


\section{Dompter la mer sauvage réflexions sur Evryali de lannis Xenakis}

Marc Couroux

Evryali de lannis Xenakis, composé en 1973, marque un point tournant dans l'histoire du répertoire pianistique et de l'« interprète-médium ». Antérieurement, malgré la difficulté croissante des œuvres pianistiques (en raison des progrès en "science performative»), il était toutefois possible à quelque virtuose de les réaliser pleinement, au moins au plan technique. À cause de certains phénomènes irréductibles, Evryali contient des passages physiquement irréalisables. Cet article explorera le problème de l'impossibilité et offrira des solutions (provisoires) qui m'ont permis de contrôler Evryali avant de l'interpréter en public.

\section{Deux prémisses techniques: le contrepoint arborescent...}

La source de cette impossibilité se situe au niveau des techniques compositionnelles de Xenakis, en particulier celle des arborescences: des dessins en forme de branches tracées sur du papier graphique, traduits ensuite en notation musicale. (L'axe des $x$ représente le temps, l'axe des y représente la hauteur des sons. Cf. exemple 1.)

Xenakis utilisa cette technique depuis le concerto pour piano SynaphaiConnexities de 1969, dans lequel la partie de piano est notée sur un maximum de dix portées, chacune composée d'une ligne différemment articulée et extrêmement directionnelle (cf. exemple 2). En effet, cette musique possède un contrepoint très raffiné, non thématique, réduit à son essence linéaire, soit la vitesse et la direction des lignes. La confluence de toutes ses 
Exemple 1

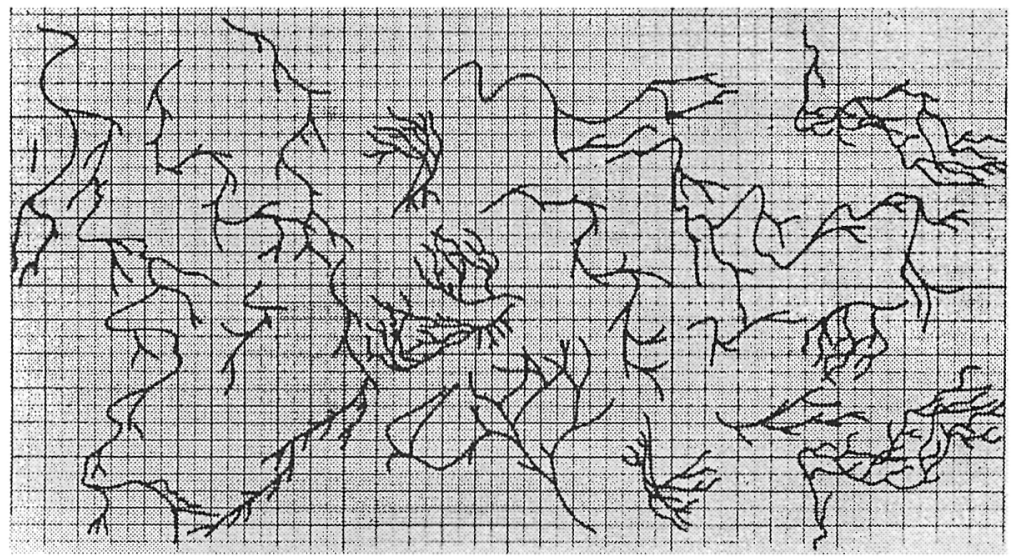

Exemple 2

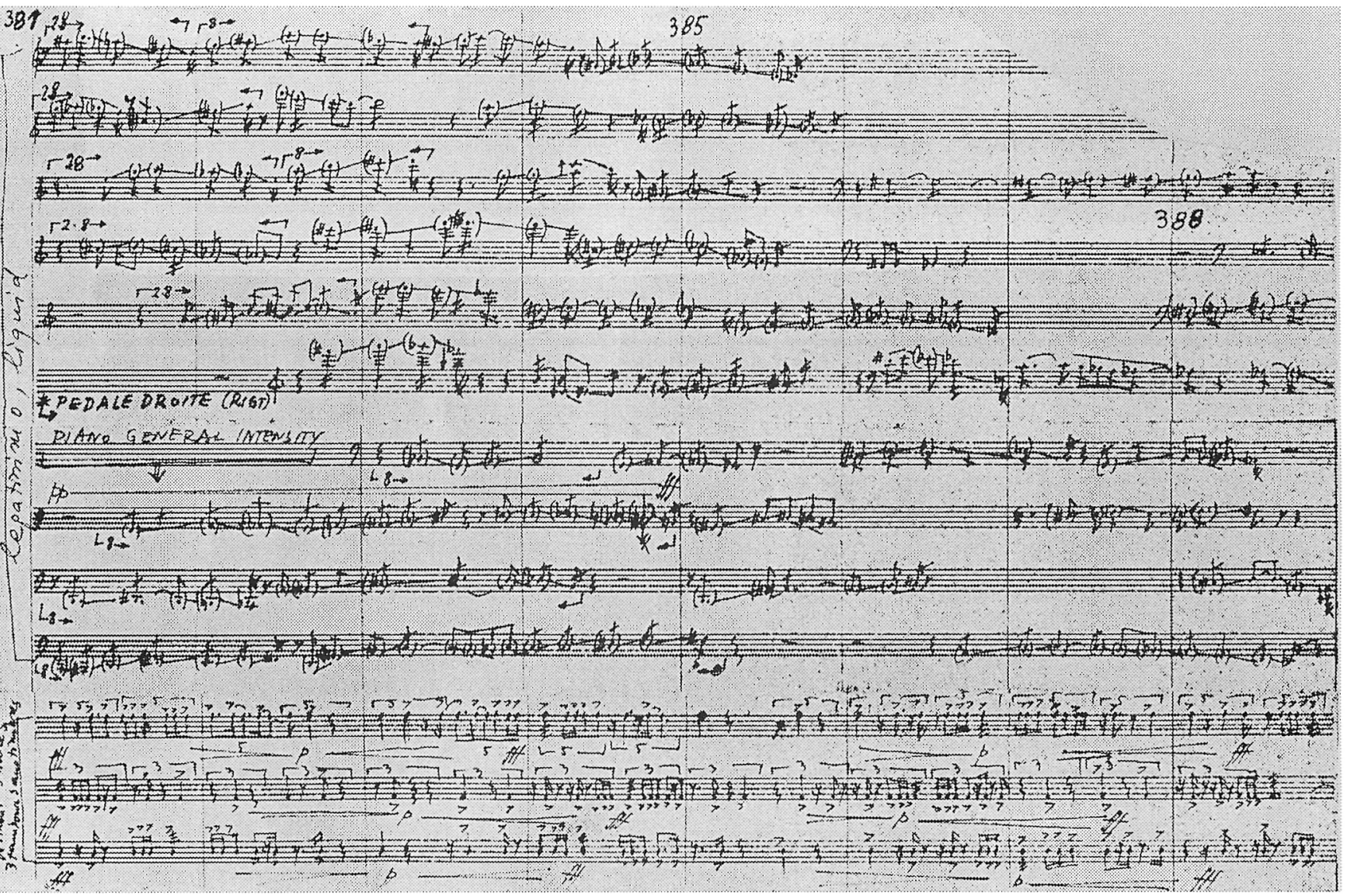


voix produit une texture très complexe. Par la suite, au sein d'œuvres telles que Phlegra (1979) et Palimpsest (1979), Xenakis utilise un contrepoint plus traditionnel, plus mélodique, défini par les gammes non-octaviantes, ayant un profil thématique distinct.

Un exemple classique de contrepoint arborescent se trouve à la page 9, où une ligne initiale finit par se multiplier par cing (cf. exemples 3 et 4). Le problème majeur de cette pièce réside en cette technique innocente - et plus spontanée que les mathématiques austères utilisées dans les années 1960. Quand les lignes se fissurent pour se propager dans diverses directions, Xenakis ne s'occupe pas de la morphologie de la main humaine, ou même du nombre (deux) de mains d'un seul interprète! $\grave{A}$ un certain moment $(X)$, la réalisation parfaite et simultanée de toutes ces lignes devient impossible par la suite (cf. exemple 4). L'interprète vient d'être confronté pour la première fois à un fait inéluctable : il ne pourra jamais donner une version intégrale de l'œuvre.

\section{... et la théorie du chaos ${ }^{(1)}$}

Dans la vaste branche scientifique qui étudie le "chaos»/science proliférante), nous rencontrons couramment la notion suivante : l'ordre contenu dans le désordre apparent/le désordre contenu dans l'ordre apparent. Un passage particulier d'Evryali illustre bien le deuxième volet de cette proposition (cf. exemple 5).

L'ordre apparent est représenté par l'accord de huit sons - en forme intégrale seulement au moment de la dernière attaque. Le désordre, par contre, est créé par la densité changeante de ce même accord à chaque attaque de doubles-croches; ni motif récurrent, ni périodicité ne s'y retrouvent. (La distribution des densités est statistiquement calculée.) Toutefois, si l'auditeur ne porte pas attention à ce détail "intérieur» - où s'il entend ce passage de loin -, il peut percevoir une entité globale, l'accord de huit sons, dont les composantes réapparaissent selon un taux assez élevé pour créer une cohésion auditive.

\begin{abstract}
(1) La théorie du chaos appliquée à Evryali n'est qu'une métaphore personnelle qui ne représente pas directement l'attitude compositionnelle de Xenakis, bien qu'il ne la désapprouverait probablement pas.
\end{abstract}

\section{Éthique}

Les territoires du compositeur et de l'interprète ne s'excluent pas mutuellement, pensons seulement à Michael Finnissy et Frederic Rzewski (deux compositeurs-pianistes poursuivant la tradition lisztiennel, mais il semble que le 
Exemple 3

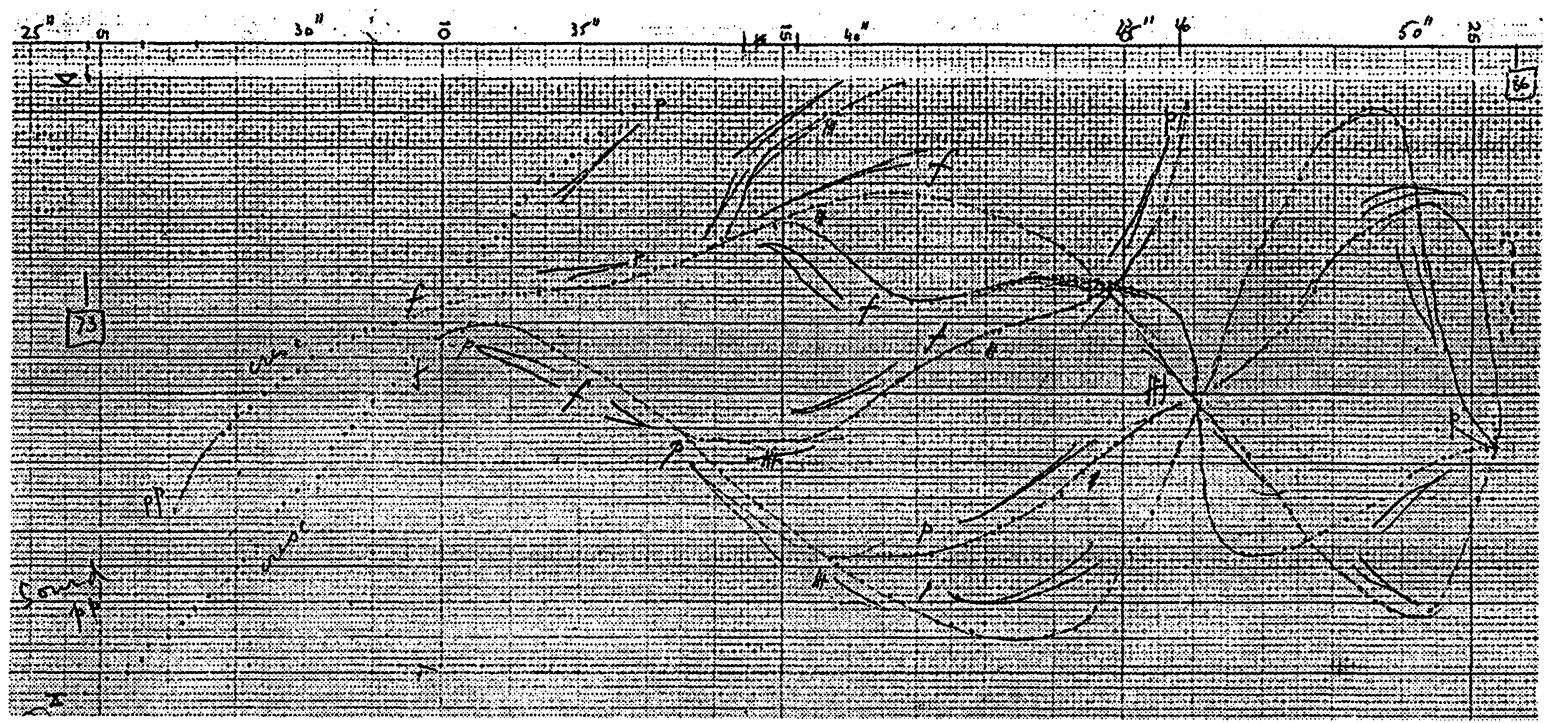

Exemple 4
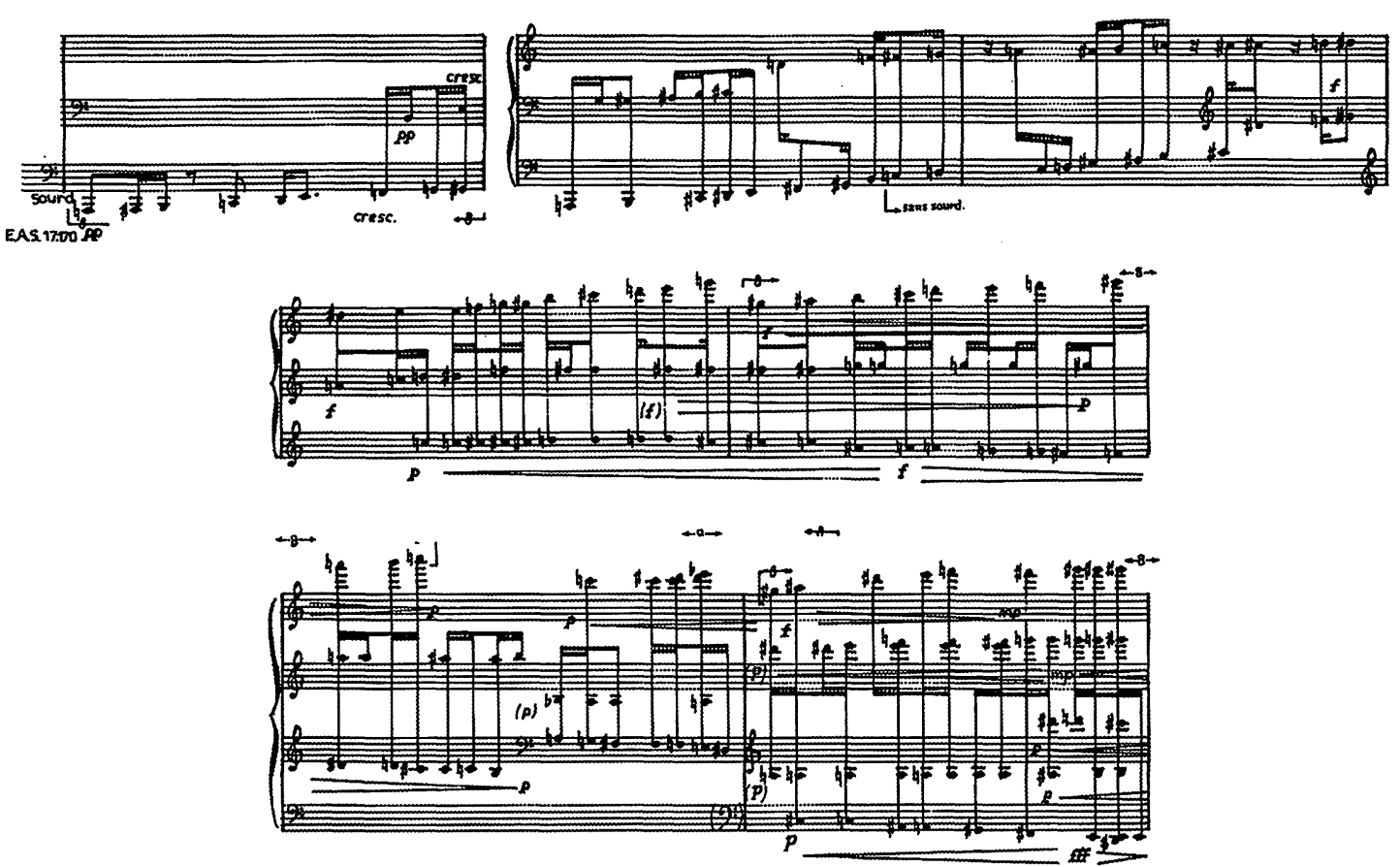
59

Exemple 5
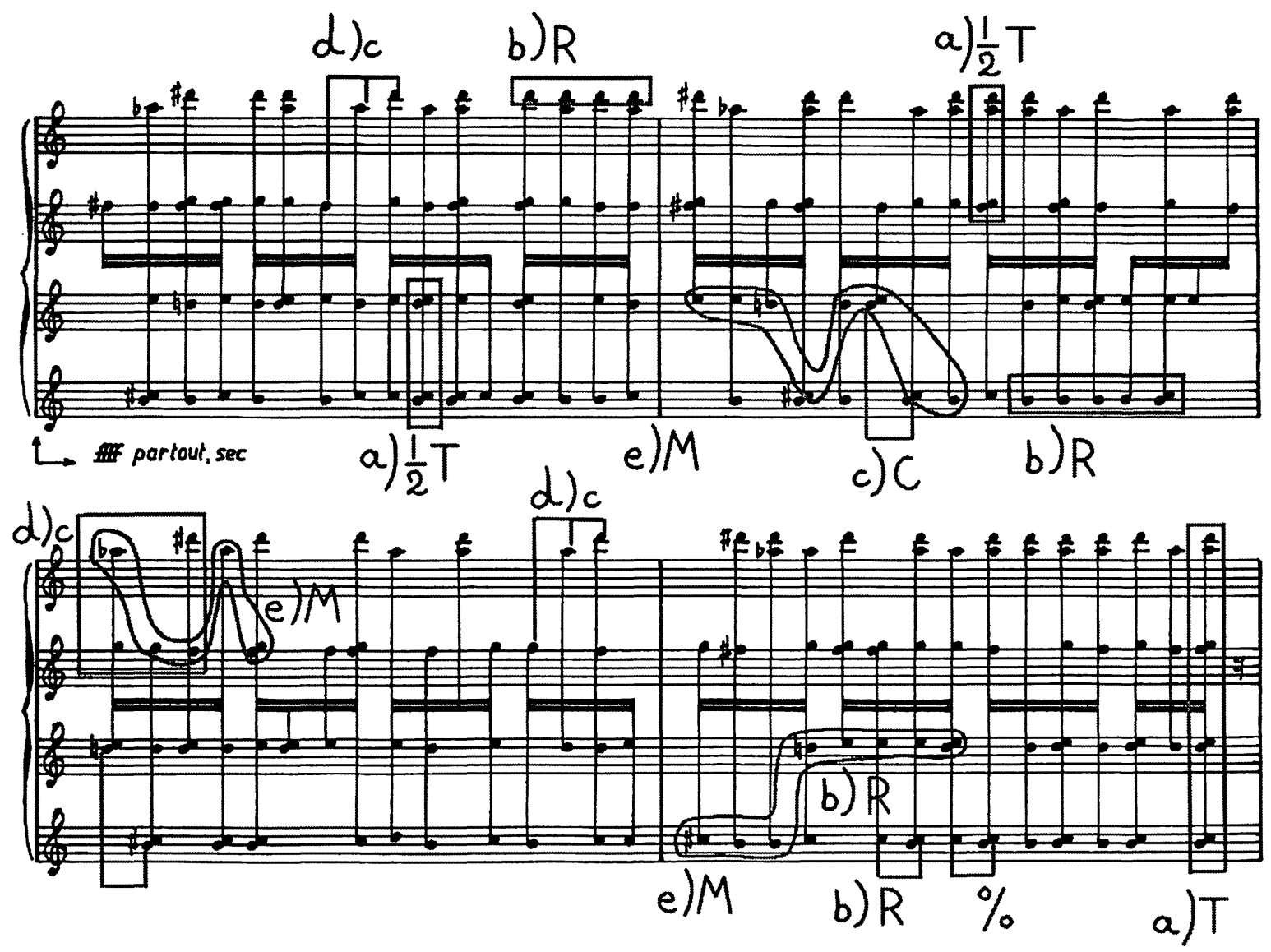

compositeur "pragmatique» soit en voie de disparition. Toutefois, est-il forcément mauvais de bousculer l'interprète dans un terrain qui lui est étranger et de remettre en question la perception de l'instrument?

Fondamentalement, l'interprète est le médium à travers lequel une œuvre d'art est canalisée (pourvu que l'on croie encore à la pertinence d'interprètes humains dans cette ère de réalisations informatiques). Sa responsabilité est impérative face aux problématiques soumises par le compositeur, il se doit de façonner les abstractions en matière sonore tangible.

Le respect absolu du texte musical est une valeur des plus inébranlables, inculquée sans répit à l' «interprète-étudiant», les fausses notes n'étant rien de moins que des péchés capitaux. Malheureusement, cette valeur ne peut plus être respectée dans Evryali, à moins que l'interprète ne démissionne let ne joue pas la piècel, préférant garder à l'esprit l'image originelle et non 
corrompue de l'œuvre. Mais l'aspect «chaotique» d'Evryali peut le guider hors de sa crise existentielle vers une conception du résultat global. Dans le contexte de ce contrepoint radical, qui ne dépend pas des hauteurs exactes pour être saisi et compris, de petites erreurs de notes perdent de leur importance. Plutôt - bien qu'on ne doive pas renoncer à une exactitude acharnée même si elle est foncièrement décevante -, on se doit de maintenir l'effet global. Par exemple, les dynamiques et l'utilisation de la pédale donnent une perspective aux masses mouvantes. (cf. exemple 6).

Xenakis a souvent comparé l'interprète à un athlète qui s'efforce toujours de se dépasser. Il a fait sien cet état d'esprit dans Evryali en créant des situations qui ne pourront jamais être réalisées. L'interprète doit entretenir une attitude positive et ouverte qui le mènera peut-être éventuellement à la perfection, sachant simultanément que c'est illusoire. Mais c'est cette conscience paradoxale qui investit Evryali de tant d'énergie.

Exemple 6
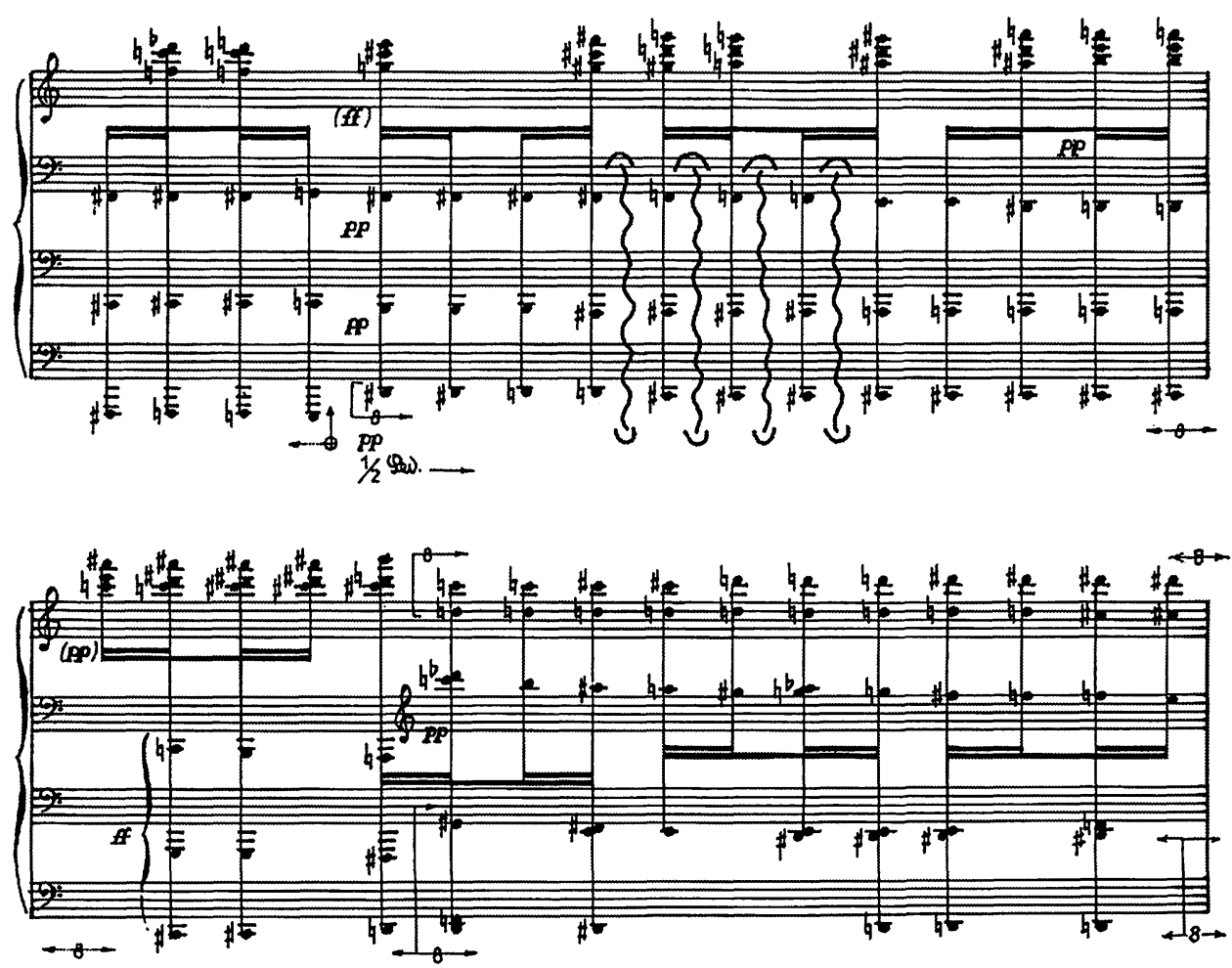


\section{Problèmes pratiques et quelques solutions}

Une controverse surgit en 1975 dans les pages de Tempo, périodique anglais de musique contemporaine. Peter Hill (1975), pianiste anglais, écrivit un article destiné à démystifier "finalement» l'aspect pratique d'Evryali. Les pianistes Yuji Takahashi (1975) et Stephen Pruslin, tous deux interprètes d'Evryali, ont répliqué à cet article dans le même périodique, critiquant Hill pour ce qu'ils appelaient ses échappatoires. Hill répliqua, en accusant Takahashi d'avoir perdu contact avec la réalité. Cette controverse explique ce qui suivra.

Hill suggéra de "réduire» certaines sections /notées sur quatre ou cinq portées) sur les deux portées traditionnelles afin de faciliter l'apprentissage. Cela revient (aussi bête que cela puisse sembler) à faire une réduction de piano d'une pièce pour piano (cf. exemple 7)! L'idée est séduisante lie l'ai moi-même essayée au départ), car elle rend explicite ce qui ne peut pas être accompli à deux mains. Mais cette simple renotation soulève certains problèmes. D'abord, quoique la notation sur quatre portées ne soit pas facile à lire (et à apprendre), elle rend évident l'aspect linéo-contrapuntique d'Evryali (cf. exemple 8/ plutôt que l'aspect harmonique devenu proéminent dans la réduction. C'est de cette façon que le compositeur a voulu "présenter "son œuvre à l'interprète, et même s'il ne peut plus contrôler ce que l'on fait avec son texte, je pense que la notation elle-même a le pouvoir d'influencer psychologiquement l'interprète. Les raffinements de notation, aussi déconnectés qu'ils soient d'une réalité interprétative, contribuent à la puissance d'une œuvre. L'interprète doit tenir compte de l'ensemble d'informations fournies, et non pas les distorsionner pour mieux servir ses besoins perceptuels. Doiton encore rappeler à l'interprète qu'il est bel et bien l'esclave de l'œuvre?

Plus qu'une simple transcription mécanique, le processus d'arrangement implique un cheminement "choisi » prédéterminé et fixé pour toujours par écrit (cf. exemple 9). Comme dans la réduction d'orchestre traditionnelle, les parties jugées superflues sont sacrifiées afin de permettre à un seul pianiste de réaliser l'essentiel. Inévitablement, dans Evryali, le pianiste doit choisir un chemin compte tenu des impossibilités, pour ensuite pouvoir l'interpréter en public sans se réfugier dans l'improvisation incontrôlable. Ce qui dérange dans le propos de Hill, c'est son aspect irréversible, car une version qui correspond aux capacités de l'interprète au moment même du choix ne tient pas compte de son futur potentiel, lorsqu'il aura acquis la virtuosité nécessaire pour ajouter des notes. Il devrait toujours avoir la chance de revoir ses "priorités" qui permettraient une réalisation adéquate de l'effet global (créer l'illusion de plusieurs voix). La question inévitable se pose alors: serait-il possible de 
Exemple 7

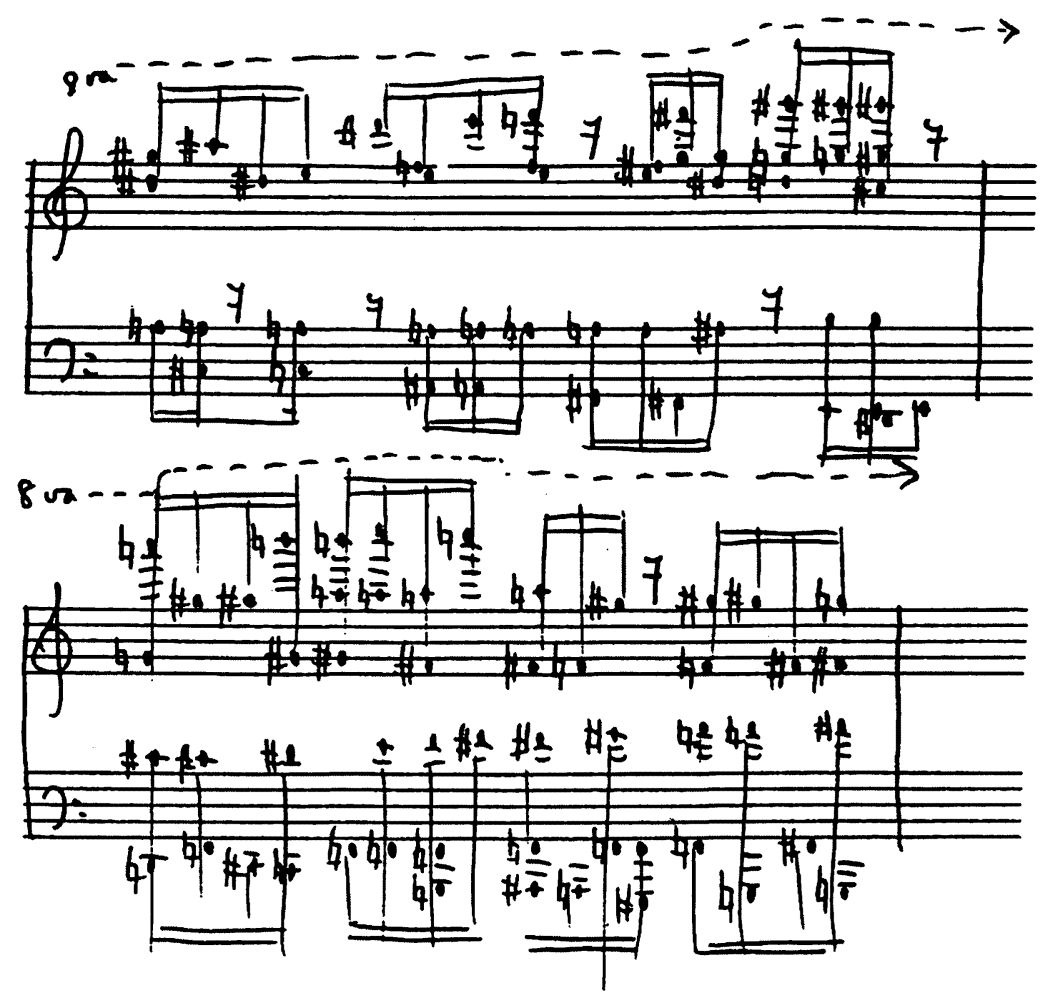

Exemple 8

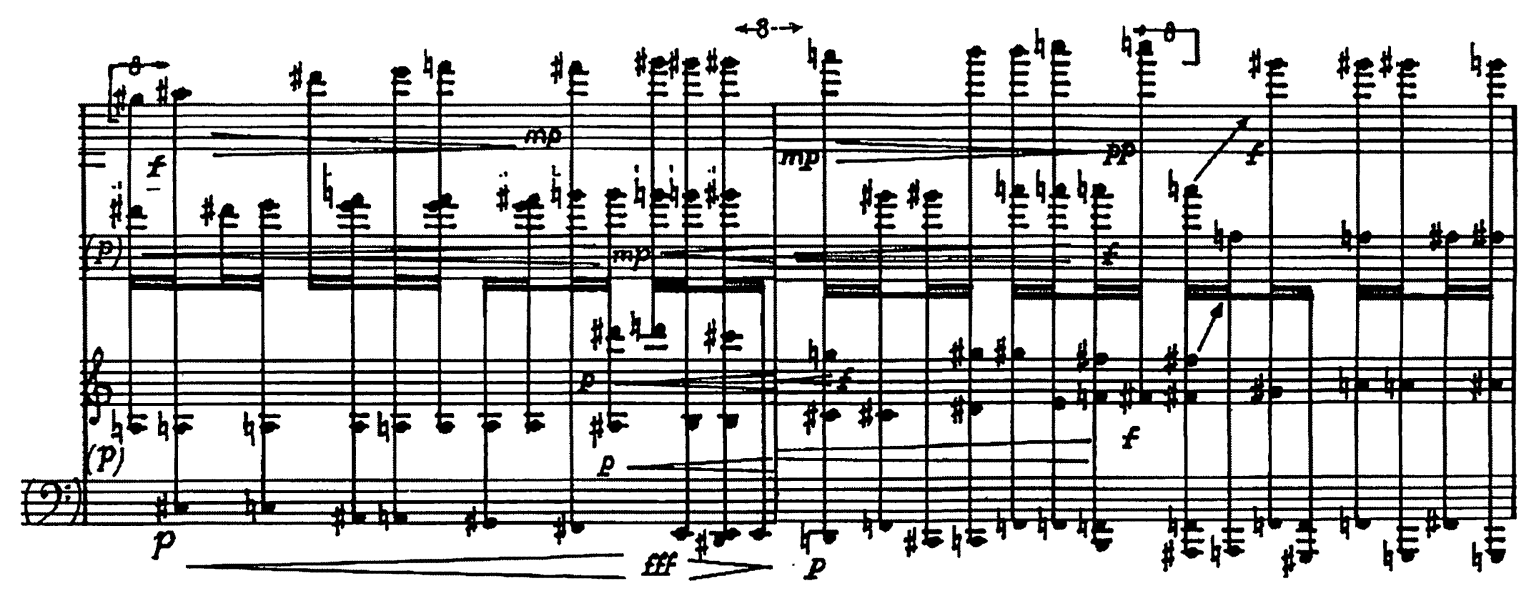


Exemple 9
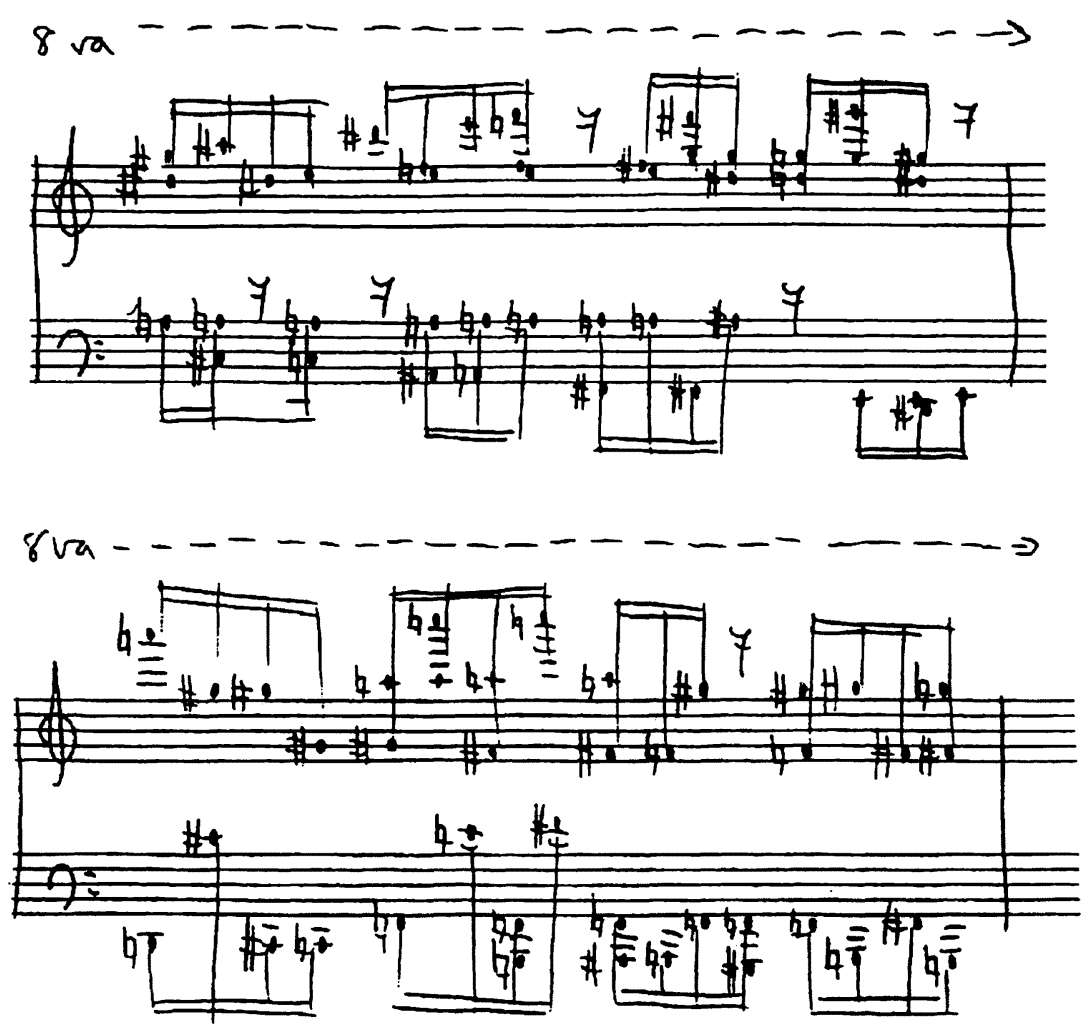

réaliser cent pour cent de la partition après dix ans de travail, ou est-ce qu'Evryali a définitivement franchi une frontière qu'aucun pianiste futur ne pourra traverser?

Hill propose ensuite carrément de réécrire certaines sections pour satisfaire les capacités physiques de l'interprète; pour aussi le sécuriser face au gouffre de l'impossible - rendre le passage soudainement possible. II propose de réduire l'empan de certains accords pour les rendre accessibles à une main humaine, entassant ainsi le contrepoint dans un espace plus étroit /donc moins perceptible de par la proximité du registre). De plus, il transpose librement certaines notes et lignes à l'octave (plus haut ou plus bas), favorisant ainsi la "classe des hauteurs" plus que la perception de lignes. Hill semble avoir mal compris l'inspiration même d'Evryali (les arborescences), car cette transposition ad libitum détruit le mouvement contrapuntique. Takahashi l'a bien dit dans sa réplique: "Evryali n'est pas une étude dodécaphonique». (1975, p. 53.) 


\section{Définir ses priorités : le tempo}

Au fond, il s'agit de décider quels aspects d'Evryali sont essentiels ef doivent être sauvegardés, malgré le sacrifice de certains autres détails. Chaque interprète doit décider. Takahashi arpège certains accords impossibles de la section centrale (cf. exemple 6), réduisant le tempo. II faut savoir que Xenakis approuva cette solution allant même jusqu'à la proposer à certains pianistes. Personnellement, j'ai choisi de maintenir l'effet de masse; j'ai alors conservé le tempo initial (blanche $=60$ ) durant toute la pièce, même si cela suppose une plus grande approximation des hauteurs. Se peutil qu'Evryali, en dépit de toutes les apparences, soit une forme d'œuvre ouverte, formellement fermée mais, par contre, assujettie aux priorités personnelles de l'interprète quant au résultat "expressif » final?

Pourtant, Evryali n'est pas une œuvre aléatoire. Tous les efforts de l'interprète doivent être canalisés vers une réalisation optimale, la partition complètement définie l'exige. Néanmoins, on doit se méfier de l'interprète qui déclare n'avoir jamais eu recours à l'improvisation dans le feu de l'action! Si l'on garde le tempo rapide, la densité de certaines sections peut devenir trop accablante pour en permettre une audition adéquate et ainsi un contrôle physique adéquat. (cf. exemple 6). Dans les sections de blocs statistiques (cf. exemple 5), Hill agit comme nous le ferions en interprétant une partition notée graphiquement, réalisée spontanément suivant la distribution générale des points $^{(2)}$. En somme, l'improvisation. Cette section est difficile à imaginer et à entendre : nous n'avons pas l'habitude d'écouter à l'intérieur des sons, comme à l'intérieur de cet accord de huit sons avec toutes ses fluctuations de densité. Je suggérerais de chercher des périodicités à l'intérieur de ce qui semble être infiniment différencié.

L'idée directrice derrière cette technique est de rendre la musique statistique accessible à la mémoire (qui n'est rien de plus qu'un muscle). J'ai ainsi élaboré un certain nombre de concepts (cf. exemple 5):

a) $T=$ total (les huit notes sont présentées verticalement, il peut y avoir un total de quatre sons pour chaque main);

b) $R=$ répétition (notes répétées qui semblent surgir de la texture);

$c$ et $d$ ) $\quad C=$ complément (main droite et main gauche traitées séparément) : ainsi deux notes sont suivies par leur complément (les deux autres) soit $\mathrm{cl}$ linéairement ou $\mathrm{d}$ ) verticalement;

e) $M=$ mélodie particulièrement mémorable.

Manifestement, la combinaison du linéaire et du vertical engendre un danger de confusion plutôt que d'accessibilité mnémonique. De plus, il est
(2) Xenakis dit (traduction libre): « Le hasard [...] peut être construit avec une grande difficulté, à partir de raisonnements complexes symbolisés par des formules mathématiques; il peut être légèrement construit, mais jamais improvisé ou imité intellectuellement. » (1972, pp. 38-39.) 
possible qu'en rendant accessibles certaines mélodies, survienne le désir d'accentuer irrégulièrement. Ceci irait à l'encontre du principe mécanique de l'attaque "stochasticcato" induite par l'esthétique des phénomènes naturels si chers à Xenakis (qui, par sa froideur, augmente la difficulté d'entendre les variations à l'intérieur de l'accord!). II est quasiment impossible de réaliser précisément ce passage (même s'il ne se pose pas de problèmes physiques), mais l'énergie intellectuelle requise pour passer au travers contribue à faire d'Evryali une bataille.

Pour créer l'illusion de jouer toutes les lignes simultanément, la solution la plus logique, quoique dangereuse, s'avère être celle qui consiste à "sauter" entre les lignes: maintenir la vie d'une ligne en la nourrissant constamment de notes. Cette solution présente certaines embûches, la plus manifeste étant la réalisation de sauts inexacts dû au tempo rapide (il est tout de même préférable de tomber autour du but que de ne pas essayer!). Aussi est-il tentant de sauter périodiquement, c'est-à-dire selon un rythme régulier (alternant deux par deux) (cf. exemple 10). Cela donne alors l'impression d'une ligne résultante extrêmement disjointe, plutôt que deux lignes chromatiquement conjointes. II faut donc sauter "apériodiquement», certes en suivant la logique générale de chaque ligne, mais aussi irrégulièrement que possible (cf. exemple 11). La tâche est moins ardue si l'on attribue une nuance dynamique particulière à chaque ligne (suivant le contexte dynamique global ainsi que le registre). Mais en dépit de ces limitations, il reste préférable de tenter de jover, plutôt que d'omettre, des lignes entières, ce qui réduirait la puissance arborescente de l'œuvre. J'utilise aussi la méthode "des crayons de couleur" de Claude Helffer (1981, p. 202), plus particulièrement pour encercler en rouge tout ce que je peux jouer de la main droite, et en bleu ce que je peux jouer de la main gauche. La possibilité d'ajouter des notes au fur et à mesure reste donc toujours ouverte.

Pour interpréter Evryali, il faut tenter de "dompter la mer sauvage». II faut imposer le maximum de contraintes physiques sur l'œuvre monstrueuse pour ensuite l'apprivoiser. Toutefois, Evryali demeure considérablement moins complexe que la «mer au large», un des sens du titre! Le mot clé reste "lucidité »: l'acceptation rationnelle de l'impossible, "interprète responsable " (comme tout guerrier à la Don Juan ${ }^{(3)}$, sans avoir recours à une improvisation non contrôlée. Il est essentiel de toujours garder à l'esprit l'extraordinaire beauté esthétique d'Evryali. L'interprète peut donc se dépasser en toute confiance, puisque cela en vaut la peine ${ }^{(4)}$. Le fait que l'on ne peut réaliser intégralement Evryali donne à cette œuvre un caractère par trop utopique. Car, même à l'intérieur des œuvres de Beethoven, nous nous efforçons d'assumer tous les détails. Le problème d'Evryali est simplement situé à un autre niveau de
(3) Les livres de Carlos Castaneda (Voyage à Ixtlan, Histoires de pouvoir) m'ont été d'une grande inspiration, durant l'apprentissage d'Evryali, surtout en ce qui concerne le devenir d'un guerrier, tel qu'enseigné par Don Juan, le sorcier-maitre de Castaneda. J'encouragerais tout futur interprète de Xenakis lou de tout autre compositeur!) à se procurer ses livres.

(4) Lors de ma rencontre avec Xenakis à Paris, en février 1994, il m'a doucement demandé « si je remettais en question l'œuvre à chaque interprétation ». Ces mots résonnent encore en moi. 
Exemple 10

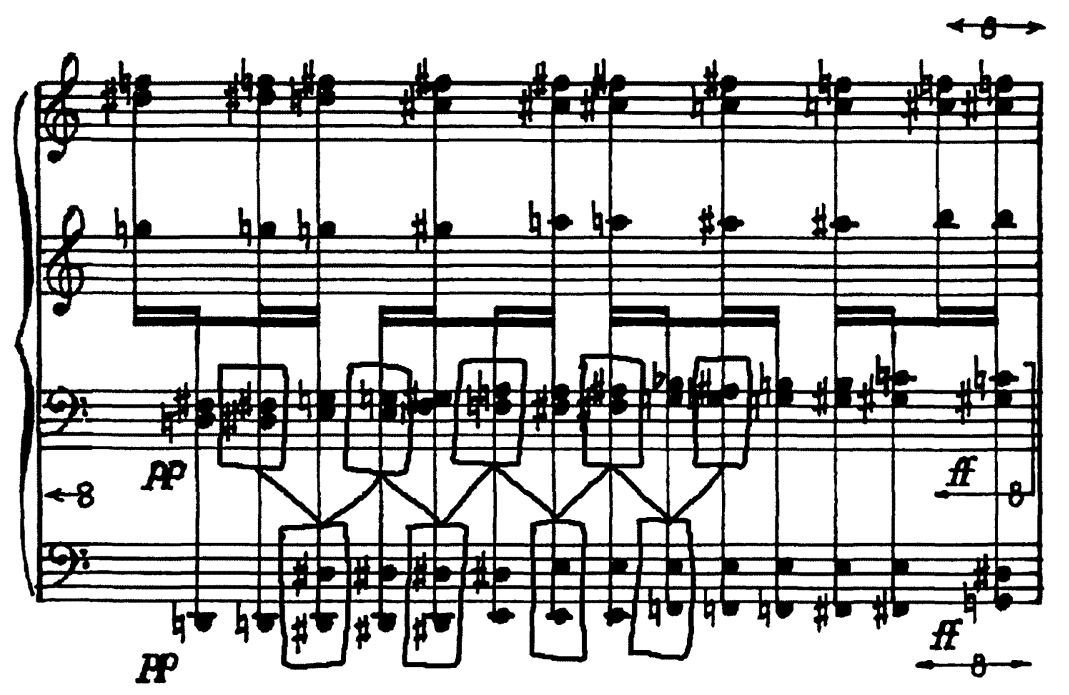

\section{Exemple 11}

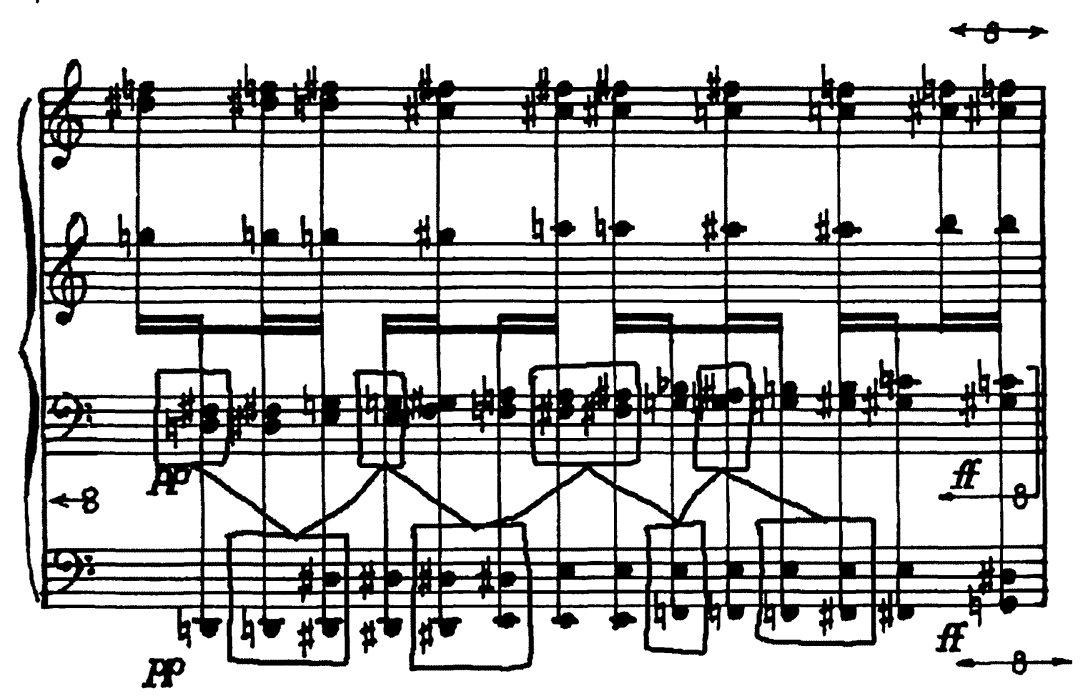


compréhension. II serait intéressant de constater l'effet que ferait cette œuvre, interprétée par un ordinateur. Aurait-elle la même tension?

HELFFER, Cl. (1981), Regards sur lannis Xenakis, "Sur Herma et autres», Paris, Stock, p. 202.

HILL, P. (1975), «Xenakis and the Performer», Tempo, $n^{\circ}$ 112, mars, pp. 17-22.

TAKAHASHI, Y. (1975), «Letters», Tempo, n 115, décembre, p. 53.

XENAKIS, I. (1972), Formalized Music: Thought and Mathematics in Composition, Bloomington, Indiana University Press. 


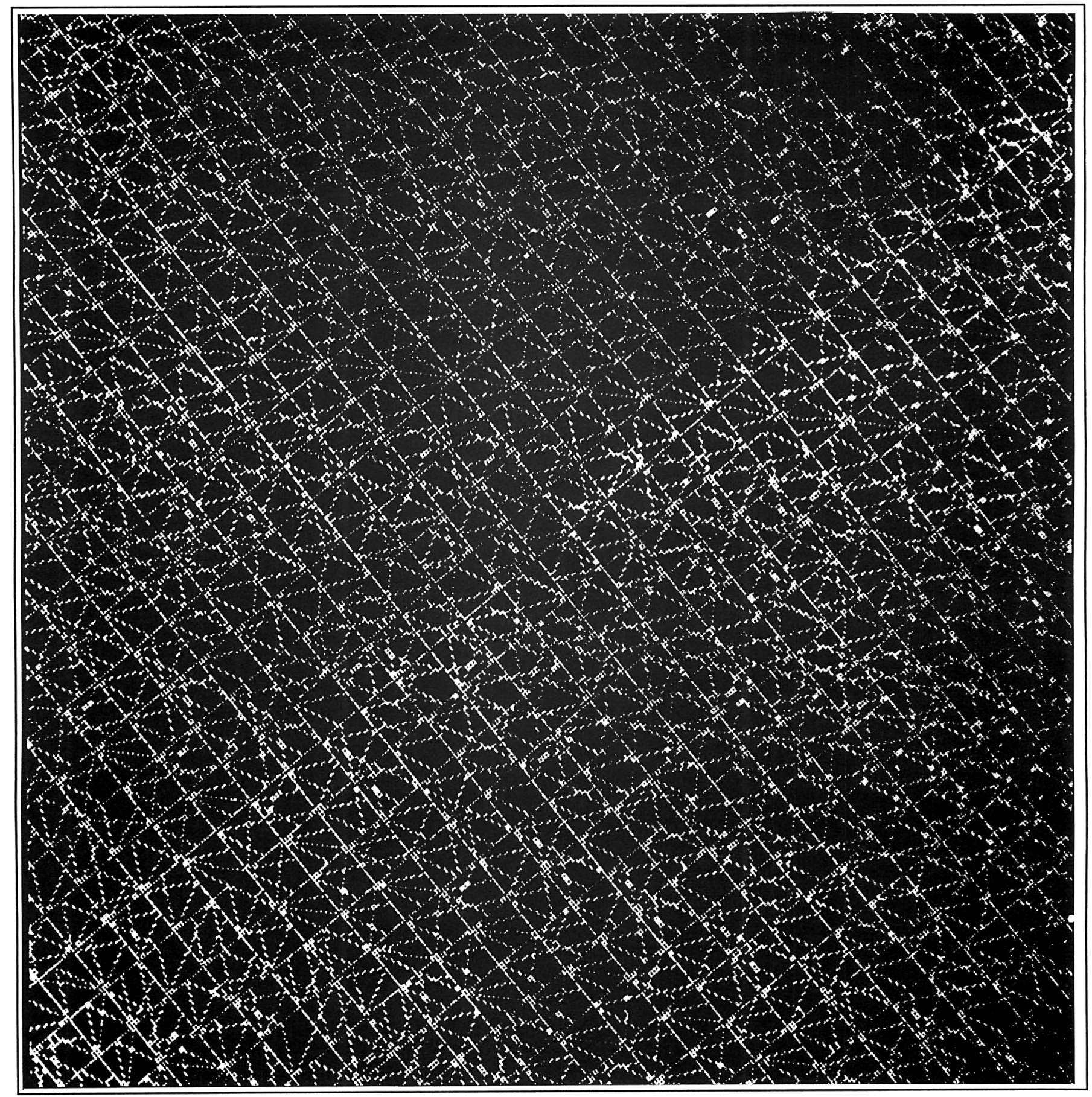

